\title{
A rare presentation of Escherichia coli urosepsis complicated by myositis
}

\author{
Ling Xu, Hua Kiat Chen
}

Department of General Medicine, Armadale Hospital, Perth, Australia

\section{CASE STUDY}

Please cite this paper as: Xu L, Chen HK. A rare presentation of Escherichia coli urosepsis complicated by myositis. AMJ 2019;12(10):273-275.

https://doi.org/10.35841/1836-1935.12.10.273-275

\section{Corresponding Author:}

Ling Xu

Department of General Medicine, Armadale Hospital, 3056

Albany Highway, Perth, Australia

Email: Ling.Xu@health.wa.gov.au

\section{ABSTRACT}

Pyomyositis is a purulent infection involving the skeletal musclesas a result of haematogenous spread. Escherichia coli (E. coli) is a rare cause for pyomyositis and previous published case reports mainly involved immunocompromised patients with haematological malignancies. We present a case of a 42-year-old immunocompetent female with confirmed $E$. coli urosepsiscomplicated by development of stage 1 pyomyositis. Early detection is essential where pyomyositis should still be suspected as a differential diagnosis in immunocompetent patients with confirmed $E$. coli bacteraemia with signs of suspected localised muscle infection.

\section{Key Words}

Escherichia coli, myositis, magnetic resonance

\section{Implications for Practice:}

\section{What is known about this subject?}

Pyomyositis is commonly caused by gram positive organism affecting immunocomprimised patients.

\section{What new information is offered in this case study?}

Infective pyomyositis can occur via haematogenous spread in immunocompetent patients with Escherichia coli bacteraemia.

3. What are the implications for research, policy, or practice?

Escherichia coli pyomyositis should be suspected as a differential diagnosis in immunocompetent patients with confirmed $E$. coli bacteraemia exhibiting signs of localised muscle infection.

\section{Background}

Infective myositis or pyomyositis is commonly caused by gram positive organisms such as Staphylococcus aureus through haematogenous spread. Due to its rare entity, infective myositis may not be suspected in immunocompetent patients with Escherichia coli bacteraemia. We present an emergent case of myositis as a complication of Escherichia coli urosepsis.

\section{Case details}

A 42-year-old woman presented with four-day history of dysuria, urinary frequency and urgency, associated with subjective chills and suprapubic pain. Significant comorbidities include hypertension and Type I diabetes.

Biochemistry tests showed elevated white cell count of $14.58 \times 109 / \mathrm{L}$ and elevated CRP of $150 \mathrm{mg} / \mathrm{L}$. Mid-stream urine cultured $E$. coli. Ultrasound kidney, ureter and bladder showed small amount of free right sided perinephric fluid without other radiological features of pyelonephritis. Patient was commenced on intravenous Amoxycillin and Gentamicin, along with other supportive treatment. After 48 hours, blood cultures were positive for Escherichia coli sensitive to both amoxicillin and gentamicin. Gentamicin was ceased and intravenous Amoxycillin was continued for a total of four days.

Patient was stepped down to oral amoxicillin but subsequently developed low grade fevers associated with new onset right lower back pain on day 5 . On clinical examination, there was localised right paraspinal tenderness at L4/L5 level with normal lower limb neurological findings. Repeat biochemistry tests showed uptrending white cell count from $10.19 \times 109 /$ L to $15.3 \times 109 /$ L. 
Patient subsequently underwent Magnetic Resonance Imaging of the lumbar spine which showed low grade oedema involving the right psoas muscle and paraspinal muscle at L4 level with low grade gadolinium enhancement, consistent with early infective myositis. No focal abscess was identified.

Following consultation with the infectious disease team, patient was re-started on intravenous antibiotics for a further 48 hours before oral stepdown to Amoxycillin for a further twelve days to complete a total course of three weeks. Patient improved with no further fever and was discharged home after inpatient stay of nine days.

In Figure 1 axial MRI imaging obtained at L4 level reviewing early infective myositis affecting the right psoas and right paraspinal muscles. No abscess formation was identified.

\section{Figure 1: Axial MRI image}

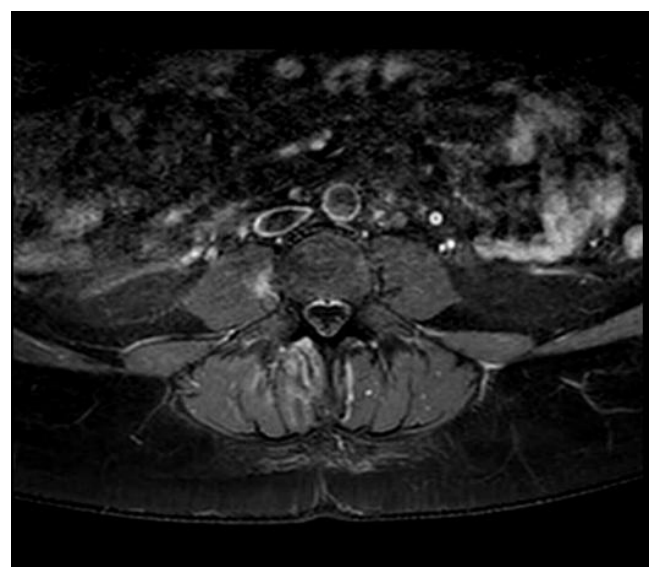

\section{Discussion}

Pyomyositis is characterised as purulent infection of skeletal muscle usually as a result of haematogenous spread. ${ }^{1}$ It was previously recognised as an infection of the tropical region, however, increasing incidence have been observed in temperate regions with associations to factors such as immunodeficiency, trauma, intravenous drug use and malnutrition. ${ }^{2-5}$ Common microbial cause of pyomyositis is Staphylococcus aureus, which has been previously reported as up to 90 per cent of cases in tropical regions and 75 per cent in temperate regions. ${ }^{2,4,6}$ Gram-negative bacterial pyomyositis remains uncommon where only seven species of Gram-negative organisms have been reported in patients with haematological malignancies: E. coli, Aeromonashydrophila, Serratiamarcescens, Salmonella sp., Klebsiella pneumoniae, Pseudomonas aeruginosa and Stenotrophomonasmaltophilia. ${ }^{7}$ To our knowledge, only 20 cases of $E$. coli pyomyositis have been previously reported where majority involved immunosuppressed patients with haematological malignancies.

The main differential diagnosis is early development of secondary ipsilateral iliopsoas abscess as a result of direct spread of infection from the adjacent kidney. Typical risk factors include diabetes mellitus, old age, recent trauma, immunosuppression, intravenous drug use, ruptured renal abscess or procedure to the inguinal, lumbar spine or hip region. $^{8-10}$ Diabetes mellitus has been long recognised as one of the predisposing factors for complicated urinary tract infections including development of renal and perinephric abscesses. $^{11,12}$ Hyperglycaemic-related impairment of immune response and vascular insufficiency are the main pathogenic processes involved for the increase risk. ${ }^{11,12}$ In a case series consist of 11 patients, diabetes was present in four out of nine patients with secondary iliopsoas abscess (44.4 per cent). From previous literatures, main pathogens identified was Staphylococcus aureus (27-88 per cent), followed by Streptococci (5 per cent) and Escherichia coli (3-18 per cent). ${ }^{13,14}$ Other case reports and small institutional series have previously reported rare cases of Klebsiella pneumoniae iliopsoas abscess from urinary tract infections. $^{15}$ However, due to the lack of radiological evidence of abscess formation, the differentiation between early secondary iliopsoas abscess and myositis cannot be made.

Diagnosis of early stages of pyomyositis remains challenging due to its subtle clinical presentation. Early symptoms include localised pain and swelling, low grade temperature with no focal abscess formation (stage 1). Fever, oedema and abscess formation (stage 2) can occur up to 10-21 days post initial onset of symptoms, follow by systemic toxicity, metastatic abscess and multiorgan failure (stage 3). ${ }^{16}$ Diagnosis can be made using $\mathrm{MRI}$ in patients with bacteraemia and suspected myositis. Treatment involves antibiotics for both $S$. aureus and gram-negative coverage, as well as surgical debridement for those with localised abscess formation. ${ }^{17,18}$ Current evidence recommends a total duration of antibiotics between three to four weeks tailored to clinical response. ${ }^{18,19}$ Although microbiology sample was unable to be obtained in this case to confirm $E$. coli as the causative organism for myositis due to absence of focal collection, patient's clinical response to antibiotics is strongly suggestive of $E$. coli myositis.

\section{Conclusion}

Although E. coli pyomyositis is uncommon, it should be suspected as a differential diagnosis in immunocompetent 
patients with confirmed $E$. coli bacteraemia exhibiting signs of localised muscle infection.

\section{References}

1. Stevens DL, Bisno AL, Chambers HF, et al. Practice guidelines for the diagnosis and management of skin and soft tissue infections: 2014 update by the infectious diseases society of America. Clin Infect Dis. 2014;59(2):147.

2. Crum NF. Bacterial pyomyositis in the United States. Am J Med. 2004;117(6):420.

3. Gibson RK, Rosenthal SJ, Lukert BP. Pyomyositis. Increasing recognition in temperate climates. Am J Med. 1984;77(4):768.

4. Chauhan S, Jain S, Varma S, et al. Tropical pyomyositis (myositis tropicans): current perspective. Postgrad Med J. 2004;80(943):267.

5. Moriarty $P$, Leung $C$, Walsh $M$, et al. Increasing pyomyositis presentations among children in Queensland, Australia. Pediatr Infect Dis J. 2015;34(1):1.

6. Small LN, Ross JJ. Tropical and temperate pyomyositis. Infect Dis Clin North Am. 2005;19(4):981.

7. Chiu SK, Chang FY. Pyomyositis caused by extendedspectrum beta-Lactamase-producing Escherichia coli in a patient with acute myeloid leukemia. Int Infect Dis J. 2009;13:85-87.

8. Buttaro M, González Della Valle A, Piccaluga F. Psoas abscess associated with infected total hip arthroplasty. J Arthroplasty. 2002;17:230.

9. Lee BB, Ngan Kee WD, Griffith JF. Vertebral osteomyelitis and psoas abscess occurring after obstetric epidural anesthesia. Reg Anesth Pain Med. 2002;27:220.

10. Yen DH, Hu SC, Tsai J, et al. Renal abscess: early diagnosis and treatment. Am J Emerg Med. 1999;17:192.

11. Fair WR, Higgins MH. Renal abscess. J Urol. 1970;104:179.

12. van den Berge $M$, de Marie $S$, Kuipers $T$, et al. Psoas abscess: report of a series and review of the literature. Neth J Med. 2005;63(10):413-416.

13. Li YC, Lin PC, Wang WS, et al. An update of Psoas muscle abscess: An 8-year experience and review of literature. Int J Gerontology. 2011;5(2):75-79.

14. Chen $Q$, Ding W, Yang D. Psoas abscess caused by actinomycete together with Escherichia coli infection: a case report and literature review. Int J clin Exp Med. 2014;7(9):2925-2927.

15. Chang CM, Ko WC, Lee HC, et al. Klebsiella pneumoniae psoas abscess: predominance in diabetic patients and grave prognosis in gas-forming cases. J Microbiol Immunol Infect. 2001;34(3):201.

16. Chiedozi LC. Pyomyositis. Review of 205 cases in 112 patients. Am J Surg. 1979;137(2):255.

17. Bickels J, Ben-Sira L, Kessler A, et al. Primary pyomyositis. J Bone JoingSurg Am. 2002:84-A:2277-86.

18. Crum NF. Bacterial pyomyositis in the United States. Am J Med. 2004;117:420-8.

19. Christin L, Sarosi GA. Pyomyositis in North America: case reports and review. Clin Infect Dis. 1992;15(4):668.

\section{PEER REVIEW}

Not commissioned. Externally peer reviewed.

\section{CONFLICTS OF INTEREST}

The authors declare that they have no competing interests.

\section{FUNDING}

None

\section{PATIENT CONSENT}

The authors, $X u L$, Chen $H K$, declare that:

1. They have obtained written, informed consent for the publication of the details relating to the patient(s) in this report.

2. All possible steps have been taken to safeguard the identity of the patient(s).

3. This submission is compliant with the requirements of local research ethics committees. 